of PS-HEMS compared to GEMS on patient outcome, primarily mortality.

Method PubMed, EMBASE, and the Cochrane Library were systematically searched on October 15, 2018 for prospective, interventional studies comparing outcomes of patients treated and/or transported by either PS-HEMS or GEMS. Outcomes of interest were mortality, time to hospital, and quality of life (QoL).

Results Of 16 studies eligible for inclusion, 12 reported on trauma patients, four on cardiac patients, and one study on stroke patients. Three studies showed significantly different mortality rates (all on trauma patients) with lower 30 day mortality $(\mathrm{OR}=0.68[0.47-0.98]$ and $\mathrm{OR}=0.41 \quad[0.2-0.87])$ and lower in-hospital mortality $(\mathrm{OR}=0.29$ [0.1-0.82]) for PSHEMS compared to GEMS. Eight studies showed significantly different time to hospital between patients transported with PS-HEMS compared to GEMS. In cardiac patients, time was significantly lower (84 vs $102 \mathrm{~min}$ and 71 vs $78 \mathrm{~min}$, both $\mathrm{p}<0.01)$. Amongst trauma patients, three showed decreased time with PS-HEMS and three showed increased time. Three studies reported QoL on trauma patients, but no benefit of PS-HEMS was found.

Conclusion In this systematic review we found a possible survival benefit for trauma patients treated and transported with PS-HEMS compared to GEMS. Furthermore, we found that time to hospital was significantly reduced in cardiac patients transported with PS-HEMS compared to GEMS.

Conflict of interest None.

Funding Departmental.

\section{HOW FIT ARE PARAMEDICS/EMTS? WHAT WE KNOW AND WHAT SHOULD BE DONE?}

C Ozkaya Senuren*, S Yaylaci. Acibadem Mehmet Ali Aydinlar University, Turkey

\subsection{6/bmjopen-2019-EMS.21}

Background Emergency Medical Systems (EMS) structures differ across the countries but there is a consensus about the importance of physical performance for the Paramedics/Emergency Medical Technicians (EMTs). However, there is no consensus about the description of their physical workload, its measurement, and evaluation. This systematic review presents the evidence for how the Paramedics/EMTs fitness levels are defined.

Method We searched the following electronic databases: Wiley Online Library, Web of Science, Scopus, Science Direct, EBSCOHost, MEDLINE, Clinical Key and Google Scholar between 2008 and 2019. Search terms related to paramedics, emergency medical technician, and physical fitness. All studies that compared physical performance outcomes included.

Results The review identified twelve full-text publications that matched the inclusion criteria (ten original research article, two systematic reviews), three of the studies investigate the physical performance demands of Paramedics/EMTs, two of them evaluate the health and physical status in current working conditions, six of them describe the risks that they have faced while performing their duties according to their performed work-related tasks and anatomical body parts.

Conclusion Physical performance of Paramedics/EMTs should be evaluated as a whole while several skills were performing simultaneously rather than individual tasks. Especially, there is a need for investigations about fine motor skills and physical performance under the acute fatigue and stress. Therefore, an easily applicable, reliable and validated 24 hour Physical Activity Assesment Questionnaire (24 hour PAAQ) should be developed which results in metabolic equivalent (MET) units to evaluate and defining physical performance requirements and criteria under every circumstance for any EMS structures.

\section{REFERENCE}

1. Fischer S. L. et al. (2017). Identifying the critical physical demanding tasks of paramedic work: Towards the development of a physical employment standard. Applied Ergonomics: 65, 233-239.

2. Coffey B. et al. (2016). A physical demands description of paramedic work in Canada. International Journal of Industrial Ergonomics: 53, 355-362.

3. Karaca, A. \& Turnagol, H. H. (2007). Reliability and validity of three different questionnaires in employees. Hacettepe Journal of Sport Sciences: 18(2), 68-84.

Conflict of interest None.

Funding None.

\section{PUBLIC AND PATIENT INVOLVEMENT IN PREHOSPITAL CARE RESEARCH DEVELOPMENT - DESIGNING THE RAPID 2 TRIAL}

${ }^{1}$ Bridie Evans*, ${ }^{1}$ J Bulger, ${ }^{2} S$ Ford, ${ }^{3} \mathrm{~T}$ Foster, ${ }^{4} \mathrm{~S}$ Goodacre, ${ }^{5} \mathrm{~S}$ Jones, ${ }^{6} \mathrm{~L}$ Keen, ${ }^{7} \mathrm{M}$ Longo, ${ }^{1} \mathrm{R}$ Lyons, ${ }^{2}$ Pallister, ${ }^{5} \mathrm{~L}$ Parry, ${ }^{6} \mathrm{~N}$ Rees, ${ }^{8} \mathrm{M}$ Robinson, ${ }^{9} \mathrm{~N}$ Siriwardena, ${ }^{1} \mathrm{~A}$ Watkins, ${ }^{1} \mathrm{H}$ Snooks. 'Swansea University Medical School, Swansea, UK; ${ }^{2}$ Abertawe Bro Morgannwg University Health Board, Port Talbot, UK; ${ }^{3}$ East of England Ambulance Service, Melbourn, UK; ${ }^{4}$ Centre for Urgent and Emergency Care Research, University of Sheffield, Sheffield, UKi ${ }^{5}$ Public and patient contributor, clo Swansea University, UK; ${ }^{6}$ Welsh Ambulance Service NHS Trust, Swansea, UK; ${ }^{7}$ School of Medicine, Cardiff University, Cardiff, UK; ${ }^{8}$ South Western Ambulance Service, Exeter, UK; ${ }^{9}$ School of Health and Social Care, University of Lincoln, Lincoln, UK

\subsection{6/bmjopen-2019-EMS.22}

Background Involving patients and public members in research helps ensure evidence is relevant, accountable and high quality. Public and patient involvement (PPI) is required in many funding applications. We aimed to involve public contributors in designing a research bid about prehospital management for hip fracture.

Method We recruited two public contributors with experience of hip fracture and prehospital care to our research team of academic, clinical and managerial partners developing the RAPID 2 proposal evaluating paramedic administration of Fascia Iliaca Compartment Block, a local anesthetic injection into the hip. We supported them to consult with a public/patient group and identify patient priorities to inform our decisions. We held research development meetings and shared project drafts to gain views, share decisions and amend documents.

Results Consultation responses suggested patient priorities after hip fracture were to return home, recover mobility and gain independence. These views guided our decisions on setting primary outcomes which were length-of-hospital-stay and health-related quality-of-life. Their concern about the study design causing delayed access to treatment meant we decided to identify common exclusion criteria before randomisation to expedite access to pain management and reduce attrition. Public contributors also agreed patients should be offered an incentive for completing and returning questionnaires to enhance data completeness.

Conclusion Involving public contributors enabled the research team to identify patient-prioritised outcomes and adjust the proposed study design to reflect these in the proposal. Public contributors will remain involved if funding is awarded to 